\title{
Creation of a Reference Image with Monte Carlo Simulations for Online EPID Verification of Daily Patient Setup
}

\author{
M. A. Descalle, C. F. Chuang, J. Pouliot
}

This article was submitted to

SPIE Medical Imaging 2002, San Diego, CA, February 23-28, 2002

U.S. Department of Energy

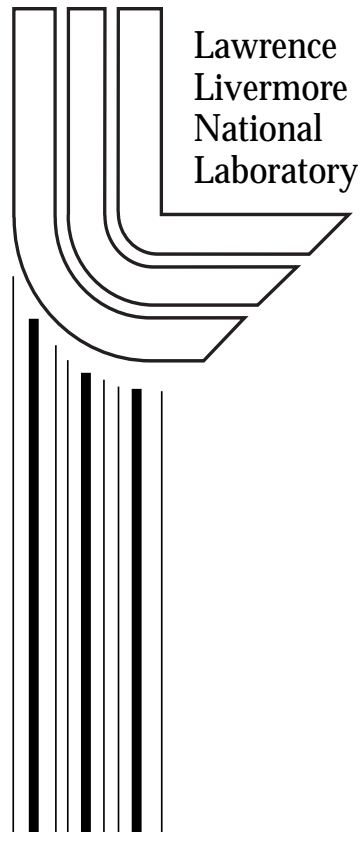

Janurary 30, 2002 


\section{DISCLAIMER}

This document was prepared as an account of work sponsored by an agency of the United States Government. Neither the United States Government nor the University of California nor any of their employees, makes any warranty, express or implied, or assumes any legal liability or responsibility for the accuracy, completeness, or usefulness of any information, apparatus, product, or process disclosed, or represents that its use would not infringe privately owned rights. Reference herein to any specific commercial product, process, or service by trade name, trademark, manufacturer, or otherwise, does not necessarily constitute or imply its endorsement, recommendation, or favoring by the United States Government or the University of California. The views and opinions of authors expressed herein do not necessarily state or reflect those of the United States Government or the University of California, and shall not be used for advertising or product endorsement purposes.

This is a preprint of a paper intended for publication in a journal or proceedings. Since changes may be made before publication, this preprint is made available with the understanding that it will not be cited or reproduced without the permission of the author.

This report has been reproduced directly from the best available copy.

Available to DOE and DOE contractors from the Office of Scientific and Technical Information

P.O. Box 62, Oak Ridge, TN 37831

Prices available from (423) 576-8401

http://apollo.osti.gov/bridge/

Available to the public from the National Technical Information Service

U.S. Department of Commerce 5285 Port Royal Rd., Springfield, VA 22161

http://www.ntis.gov/

OR

Lawrence Livermore National Laboratory

Technical Information Department's Digital Library

http://www.llnl.gov/tid/Library.html 


\title{
Creation of a reference image with Monte Carlo simulations for online EPID verification of daily patient setup
}

\author{
Marie-Anne Descalle ${ }^{\mathrm{a}}$, Cynthia F. Chuang ${ }^{\mathrm{b}}$, Jean Pouliot ${ }^{\mathrm{b}}$ \\ ${ }^{a}$ Lawrence Livermore National Laboratory, Livermore, CA, ${ }^{b}$ University of California San Francisco, \\ San Francisco, CA
}

\begin{abstract}
Patient positioning accuracy remains an issue for external beam radiotherapy. Currently, kilovoltage verification images are used as reference by clinicians to compare the actual patient treatment position with the planned position. These images are qualitatively different from treatment-time megavoltage portal images. This study will investigate the feasibility of using PEREGRINE, a 3D Monte Carlo calculation engine, to create reference images for portal image comparisons. Portal images were acquired using an amorphous-silicon flat-panel EPID for 1) the head and pelvic sections of an anthropomorphic phantom with $7-8 \mathrm{~mm}$ displacements applied, and 2) a prostate patient on five treatment days. Planning CT scans were used to generate simulated reference images with PEREGRINE. A correlation algorithm quantified the setup deviations between simulated and portal images. Monte Carlo simulated images exhibit similar qualities to portal images, the phantom slabs appear clearly. Initial positioning differences and applied displacements were detected and quantified. We find that images simulated with Monte Carlo methods can be used as reference images to detect and quantify set-up errors during treatment.

Keywords: Monte Carlo simulations, portal imaging, image correlation, reference image, set-up error
\end{abstract}

\section{INTRODUCTION}

External beam radiation therapy benefits from current advances in 3D imaging techniques. Patient 3D anatomy can now be defined by X-ray Computed Tomography (CT), and tumor delineation is greatly improved by Magnetic Resonance Imaging (MRI) tissue differentiation properties. Better tumor delineation allows for decreased margins imposed around the tumor during the planning phase. Improved patient immobilization allows for less margins than were traditionally added to account for organ motion, patient motion and set-up deviation that often occur during the length of the treatment. Tighter margins combined with the development of highly conformal treatment techniques impose stricter acceptance limits on patient positioning during treatment and a need for rapid online daily checks.

The development of electronic portal imaging devices (EPID) is critical to achieve this goal. [1,2] The new generation of amorphous silicon and selenium flat panel portal imagers addresses some of the limitations of earlier detectors such as liquid filled ion chambers and video-based systems. The new flat panels offer little or no geometric distortion, high spatial resolution and quantum efficiency, and better signal to noise ratios. Also, dose response is linear in the energy range of interest.

These characteristics should improve patient positioning accuracy during external beam radiotherapy. However, a major issue remains: the choice of a reference image. Patient treatment plans are formulated based on CT scans recorded prior to the start of treatment. Once a treatment plan has been selected, the patient is "simulated" in the treatment position with either a physical or digitally-reconstructed kilovoltage diagnostic x-ray beam, establishing the projected anatomical views anticipated for each treatment beam. During treatment, megavoltage treatment beams are then used to generate corresponding transmission images of the patient. By comparing planning-time kilovoltage radiographs with treatment-time megavoltage images, the clinician compares the actual patient position with the position that was used to plan the patient's treatment. [3] The problem is that planning images are qualitatively different from the portal images acquired during treatment because of 
the significant difference in beam energy. Diagnostic x-rays interact with tissue via photoelectric effect while the Compton effect dominates megavoltage beam photon interactions.

Beside the qualitative difference, the question remains of what should be an appropriate reference point to relate planning and treatment time positions. Three types of reference images are or could be used: X-ray films acquired during simulation, the first portal image recorded at treatment time (either film or EPID), and images/information based on the planning CT scans.

Simulation films represent an intermediary position, and one has to make the assumption that the simulation position is the same as the planning one. When the portal image acquired on the first day of treatment is used as the reference image, daily set-up deviations from that initial position are detected by automated techniques during the course of the therapy. However, this process still does not provide a direct comparison between the patient position at planning time and the position on the first day of the treatment.

Finally, information derived from the planning CT can be used as reference, such as Digitally-Reconstructed Radiographs (DRR), CT contours overlaid on ultrasound images, gold seed localization and Monte Carlo calculated images. Unfortunately, DRRs do not match the quality of films and CT to ultrasound images coregistration is complex. One option would be to use kilovoltage treatment time images for comparison, several groups are working on the development of tomotherapy or dual beam machines [4-6]. Another option is to simulate megavoltage portal images at planning time with Monte Carlo methods.

Monte Carlo methods are accurate statistical simulation methods, based on first principles, that are ideal to simulate particle transport in complex geometry. Several research groups have implemented Monte Carlo dose calculation engines for external beam radiation therapy treatment planning [7-13]. Portal imaging applications are focused on quantifying the patient scatter component, specifying detector response, and exit dosimetry [14-22]. Previously, we demonstrated that these methods could be used to generate portal images of simple phantoms. [23]

The purpose of this study is to assess the value of using Monte Carlo generated megavoltage reference images to check patient position.

\section{MATERIAL AND METHODS}

\subsection{Clinical images}

All portal images were acquired at the UCSF Comprehensive Cancer Center (San Francisco, CA) using a Siemens Primus accelerator (Siemens Oncology Systems, Concord, CA) equipped with an amorphous silicon flat-panel electronic portal imaging device (EPID) of $1024 \times 1024$ pixels resolution, $41 \times 41 \mathrm{~cm}^{2}$, and $0.400 \mathrm{~mm}$ pitch. Images are digitized over 16 bits. [24] The source to isocenter distance is $100 \mathrm{~cm}$ and the distance from the source to the detector surface is $133 \mathrm{~cm}$. We used the system in two acquisition modes: a beam pulse triggered mode that allows the acquisition of low monitor unit (MU) images (less than $2 \mathrm{MU}$ ), and a fluoroscopic mode where an image is formed by averaging a large number of frames. Images obtained in this mode are typically acquired with $50 \mathrm{MU}$ at a dose rate of $300 \mathrm{MU} /$ minute with a beam energy of $6 \mathrm{MV}$.

We used the head and pelvic sections of an anthropomorphic (Rando) phantom. These phantoms are made of slabs of soft tissue equivalent material in which a skeleton is embedded. Air gaps between the slabs create image artifacts in the CT scans, and appear clearly on portal images acquired with the amorphous silicon EPID. The implications of these air gaps for our simulations will be discussed later.

Open-field portal images were acquired with the phantoms placed at the origin. The head Rando phantom was imaged with a $15 \times 15 \mathrm{~cm}^{2}$ open field and a left lateral beam, while the pelvic region of the Rando phantom was imaged with a $20 \times 15 \mathrm{~cm}^{2}$ open field and an anterior-posterior beam. These field sizes and beam directions are typical of clinical verification images for pelvic and head treatment plans. The phantom was then moved $7-8 \mathrm{~mm}$ from the origin, in the anterior and caudal direction (head phantom), and left lateral, right lateral and caudal direction (pelvis phantom) as described in Table 1.

The Rando phantoms sections were scanned on a Siemens Emotion CT scanner. The head Rando phantom was scanned with $1 \mathrm{~mm}$ slice thickness, while the pelvis Rando phantom was scanned using $3 \mathrm{~mm}$ slice thickness. These CT scans were used for two purposes: 1) to generate Digitally-Reconstructed Radiographs, and 2) to model the 3D "patient" geometry necessary to perform 3D Monte Carlo portal image simulations.

We did a retrospective study based on a prostate cancer patient's treatment plan (7 gantry angles with 9 different fields, $18 \mathrm{MV})$. Portal images for the left lateral beam had been recorded on five different days. 


\subsection{Monte Carlo methods}

Megavoltage portal images were simulated with the PEREGRINE system, a 3D Monte Carlo dose calculation engine developed specifically for external beam radiotherapy treatment planning (Lawrence Livermore National Laboratory, Livermore, CA). It is based on 3D Monte Carlo methods and simulates beam particle transport, i.e. photons, electrons and positrons, through the patient-dependent part of the beam delivery system and through the patient. All primary and daughter particles are simulated. The beam model and physics involved in the code are described in details elsewhere. [7, 25] The patient 3D geometry is defined by a set of CT scans. The code tallies the dose deposited in the patient, and can also simulate portal images and record energy deposition at the surface of the detector. The detector location, XY dimensions and resolution are user defined. Photons exiting the patient are ray traced to the plane of the detector, and are collected when crossing the detector surface. The basic tally is a square or rectangular pixel, and the quantity reported at the end of the calculation is energy per $\mathrm{cm}^{2}$. Two components of the exiting beam are collected and reported separately: The photons that scattered in the patient, and the photons that went through the patient without any interaction, also referred to as "primary" or unscattered component. Note that these photons may have scattered in the accelerator head and modifiers.

We simulated portal images of the two Rando phantom sections and of the left lateral beam of a prostate treatment plan. We used the phase-space file of the Siemens KD2 linear accelerator 6 and $18 \mathrm{MV}$ beams, generated with the BEAM code (NRC, Ottawa, Canada), [26] to create the PEREGRINE device file representative of the UCSF Siemens Primus accelerator. The transport geometry model was defined by CT scans of the anthropomorphic phantoms and the prostate treatment plan. We tallied the exit photon energy fluence at the surface of a portal imager located $33.0 \mathrm{~cm}$ from the isocenter. The collection grid is made of $1000 \times 10000.04 \mathrm{~cm}$ pixels or $512 \times 5120.08 \mathrm{~cm}$ pixels. The unscattered component is collected independently from the patient scatter component. Calculations were stopped when the statistical uncertainty (one standard deviation) reached $1 \%$ of the maximum energy deposited.

\subsection{Image correlation}

To illustrate the use of Monte Carlo generated images as a reference image, we applied a fully automated software for the detection of setup deviation that was developed and extensively tested on pelvic field portal images. [27] First, two binary edge images are created, then the software automatically finds the best match and determines the spatial shifts between the reference and test images.

During the first step, bone edge detection is done with the Laplacian of a Gaussian (LoG) filter, for which the impulse response is described in Equation 1.

$$
\log (y, x)=\frac{2 \sigma^{2}-\left(x^{2}+y^{2}\right)}{\sigma^{4}} e^{-\left(x^{2}+y^{2}\right) / 2 \sigma^{2}}
$$

This technique convolves the LoG with the image to find the zero-crossing distribution with a user-defined standard deviation $\sigma$, where the value chosen for sigma defines the sensitivity of the edge filter. Megavoltage portal images have poor contrast and have been shown to require greater sigma values. The result of the convolution is an "edge image" consisting of closed contours. The bone edge detection is performed independently on the reference image and on the test image.

The geometric center of the treatment field is calculated from the field edge $[28,29]$ and is the origin of the coordinate system. The correlation is done solely inside the treatment field. The two bone edge images are then correlated to determine the set-up deviation in terms of translation in the $\mathrm{X}$ and $\mathrm{Y}$ directions. (See parameters $\mathrm{a}$ and $\mathrm{b}$ in Equation 2.) Note that rotational deviations are not quantified. The result is the maximum correlation to the reference image.

$$
c(a, b)=\frac{\sum_{y=0}^{H-1 L-1} \sum_{x=0} i_{r e f}(y, x) i(y+a, x+b)}{\sqrt{\left(\sum_{y=0}^{H-1 L-1} \sum_{x=0}^{L-1} i_{r e f}(y, x)\right)\left(\sum_{y=0 x=0}^{H-1} \sum^{L-1} i(y, x)\right)}}
$$




\section{RESULTS}

\subsection{Rando phantom}

Figure 1 shows three types of image for each site: a DRR, a clinical portal image and a simulated portal image. In all three types of images, we can clearly see the sequence of air gaps and slabs constituting the phantom. The DRRs exhibit high contrast between soft tissue and bone due to a dominant photoelectric absorption by the calcium contained in bones. In the megavoltage images, the Compton interactions produced by high energy photons are dominant, and the contrast between bone and soft tissue is significantly lower than in the DRR. This is well illustrated by the pelvic images, while the head images mainly show contrast between air cavities and tissue. The quality of the Monte Carlo simulated images is similar to the portal images quality in terms of gray-scale and level of details, open field size and magnification. For the anthropomorphic head phantom, the frontal, ethmoid and nasal sinuses, mandible, and hard palate can clearly be seen at similar locations in the megavolt images. We noticed a small rotation between the two images, indicating a slight difference in setup between the time of CT scanning and the time of portal imaging, and we applied a correction of 1.6 degree to the portal images. For the pelvic section, the pubic symphysis, sacro-illiac joint and femur heads are clearly recognizable. The measured image shows that the phantom was shifted vertically and up with respect to the open field. These initial setup deviations were quantified with the correlation algorithm. For the head, they were $4.7 \mathrm{~mm}$ and $0.6 \mathrm{~mm}$ in the $\mathrm{x}$ and $\mathrm{y}$ directions, respectively, and for the pelvis, the deviations were $1.0 \mathrm{~mm}$ in $\mathrm{x}$ and $15 \mathrm{~mm}$ in $\mathrm{y}$.

Numerical results for the head and pelvis are summarized in Table 1, and graphical results are presented in Figure 2. Table 1 shows the total deviations detected in the $\mathrm{X}$ and $\mathrm{Y}$ directions. The initial set-up deviations were then used to correct our evaluation of known set-up deviations, these results are reported as corrected deviations. We had applied displacements of 7 or $8 \mathrm{~mm}$, and they were all detected. However, the difference between known and detected deviations varied from under 0.7 $\mathrm{mm}$ for unidirectional displacement, to $1 \mathrm{~mm}$ (head) and $2 \mathrm{~mm}$ (pelvis) when $\mathrm{X}$ and $\mathrm{Y}$ displacements were applied simultaneously.

\subsection{Prostate patient case}

We compared the clinical and simulated portal images for a left lateral beam shaped by a multileaf collimator (MLC) during a 7-field prostate cancer treatment (see Figure 3). These two types of images appear very different to a human observer. The field edges, the femoral head and two of the three gold seeds placed around the prostate can be easily identified in the portal image. The MLC field edges clearly show in the simulated image. However, the contrast inside the treatment field is poor compared to the clinical image. This poorer quality is probably due to the fact that prostate patient CTs were scanned at 5 $\mathrm{mm}$ intervals, thus giving less image resolution for the Monte Carlo simulation. The MLC leaf positions in the simulated image match the leaf positions in the portal images, except for the three leaves located in the field upper right corner that are pulled back in the portal images. This change in MLC positions were done at the request of the clinician to increase the field size.

Table 2 shows detected displacements in the $\mathrm{X}$ and $\mathrm{Y}$ directions for five portal images of the left lateral beam, taken on five different days of treatment. In all cases, the simulated image was used as the reference for the correlation. The total detected deviations are between 8.0 and $12 \mathrm{~mm}$ in the $\mathrm{X}$ direction, and between 0.0 and $2.0 \mathrm{~mm}$ in the $\mathrm{Y}$ direction. Graphical results are presented in Figure 3.a and 3.b, for two of these portal images. In the first one, the femoral head is centered in the field, while in the second one, it shows in the lower part of the field. Despite the simulated image apparent lack of information, the femoral head contours are detected by the LoG filter, and the correlation algorithm can calculate the necessary deviations to overlap the bone edges images.

\section{DISCUSSION}

\subsection{Rando phantom}

Our results show that portal images of the anthropomorphic phantom sections can be simulated with Monte Carlo methods, using the planning CT scans. These open field images display the characteristic low contrast of images generated with a megavoltage X-ray beam, and we can identify most anatomical features present in the measured images. On one hand, the two Rando head images have a lot of details in addition to showing air gaps and slabs, and they are easy to understand. On the other hand, images of the pelvic region show relatively few details and poor contrast, since the beam goes through a larger volume/thickness of tissue, and the region of interest is essentially made of soft tissue. The vertical shift was detectable in the first portal image (no displacement), based on the femoral heads location with respect to the image horizontal central axis. However, the "slab effect" is the main observable feature of portal and simulated images. 
The portal image resolution seems to be better although we used the same detector resolution and pixel size in the simulation. It is probably due to CT scanner finite spacing. CT scans are transverse images, and the beam is perpendicular to the patient inferior-superior axis during portal imaging. When a 3D model of the patient is built, the $\mathrm{CT}$ scans are given a thickness to obtain a regular voxel transport grid. Assuming the CT-based voxel dimensions are $0.1 \mathrm{~mm}$ by $0.1 \mathrm{~mm}$ in the plane of the CT scan, and $3 \mathrm{~mm}$ in the direction perpendicular to the CT scan, when projected at the EPID surface, it translates into $0.1 \mathrm{~mm}$ along the horizontal axis (X) and $3 \mathrm{~mm}$ along the vertical axis $(\mathrm{Y})$ of the detector. During the PEREGRINE simulation, the detector pitch is set to $0.4 \mathrm{~mm}$ which images the $\mathrm{CT}$ scan slices, and yet captures details along X.

The simulated images can only be as good as the planning CT scans, and in the case of the Rando phantom, the details were sufficiently resolved to use the PEREGRINE images references for the correlation. Once corrected for initial set-up errors, the 7 to $8 \mathrm{~mm}$ displacements were detected and reported as greater than $6 \mathrm{~mm}$. Bidirectional displacements lead to greater differences in detected deviation than when we moved the phantoms solely along the $\mathrm{X}$ axis of the detector. As discussed above, the simulated image contains more details along $\mathrm{X}$ and should be more sensitive to $\mathrm{X}$ deviations, while vertical deviations are the limiting factor.

It is interesting to note that binary edge images are dominated by air gap edges. (See Figure 2.) The maximum correlation is obtained when air gap edges are overlaid. For the pelvic region, we would have expected to observe incorrect matching when large vertical displacements bring different air gaps close together, regardless of the fact that they may correspond to different air gaps within the Rando phantom. This could have lead to inconsistent results when trying to detect setup deviations, but the images were nicely overlapped for the 0 and $8 \mathrm{~mm}$ caudal deviation.

\subsection{Prostate case}

This is a stress case to test how well the simulated image acts as a reference since 1) the MLC-shaped field is relatively small compared to the open field used on the Rando phantom, 2) CT scans of the pelvic area around the prostate are usually spaced every $5 \mathrm{~mm}$ or more, and 3 ) the image contrast is poor since the beam is mainly going through soft tissue. The femoral head cannot be clearly identified and the gold seeds implanted inside the prostate appear as three black "boxy" regions. Their apparent size and shape is a consequence of the CT slice separation $(0.5 \mathrm{~mm})$ which does not resolve the length of the seeds $(3 \mathrm{~mm})$, and of the CT reconstruction algorithm artefacts in the presence of high $\mathrm{Z}$ material. It is worth mentioning that from a clinical stand point, since the prostate location varies daily depending on rectal and bladder fillings, patient positioning is based on the field edge and the three gold seeds implanted to locate the prostate. When the reference image is the first clinical image of the treatment, day to day deviations are smaller than $5 \mathrm{~mm}$ in both directions. If the simulated image is the reference image, then the deviation detected is between 8 and $12 \mathrm{~mm}$ in the $\mathrm{X}$ direction, and between 0 and $2 \mathrm{~mm}$ in the $\mathrm{Y}$ direction.

The large deviation observed between clinical images and the PEREGRINE image reflects corrections for day to day prostate movements. For the purpose of this study, we were interested in anatomical feature detection and location. As shown in Figure 3 and Table 2, the PEREGRINE image contains sufficient information to be used as a reference image for the correlation algorithm even when the femur head location varies significantly from day to day, within the treatment field. The gold seeds and the slightly different field shape do not interfere with this correlation. We observed that the MLC leaves were pulled back in the portal images to increase the field size, the PEREGRINE image allows to visualize it easily, and it gives an effective way to document that the field has been modified by the physician between planning time and treatment time.

This study seems to reveal two potential sources of errors. In the case of the Rando phantom, the dominant feature in an image may not be of clinical interest such as the air gap, and might prevent good correlation. The simulated images are sensitive to CT spacing as shown by all three cases, and can limit somewhat the detection of vertical displacement in the plane of the detector. Ideally, this parameter should be small for both treatment planning and generating the reference image.

\section{CONCLUSIONS}

The purpose of this study was to assess the value of using Monte Carlo-generated megavoltage reference images to check patient position during treatment. The novel aspect of this work is the use of planning CT scans to generate the simulated image not unlike the DRR. However, while the DRR is generated by ray tracing from the source through the patient and taking into account attenuation coefficients from CT scans, we used a realistic model of the clinical beam and a full Monte Carlo simulation to transport photon through the patient. This approach allows the comparison between planning time images and treatment time images with similar characteristics, instead of comparing kilovoltage to megavoltage images. We showed that Monte Carlo images exhibit the same characteristics as portal images for the head and pelvic regions of a Rando phantom, and can be used to test set-up error detection and quantification in the case of a prostate cancer treatment field. It seems particularly applicable to regions with numerous anatomical features such as the head and neck region. These initial 
results seem to indicate a sensitivity to CT spacing. Further studies based on clinical cases are needed to determine if this technique could be suitable to detect patient set-up errors for various cancer sites and for different treatment fields.

\section{ACKOWLEDGEMENTS}

This work was performed under the auspices of the U.S. Department of Energy by the University of California, Lawrence Livermore Laboratory under contract number W-7405-ENG-48. This work was funded in part by a grant from the DOE Office of Biological and Environmental Research (OBER).

\section{REFERENCES}

1. A. L. Boyer, L. Antonuk, M. van Herk, H. Meertens, P. L. E. Reinstein, J. Wong, "A review of electronic portal imaging devices (EPIDs)," Med. Phys. 19, 1-16, 1992.

2. P. Munro "Portal imaging technology: past, present and future," Semin. Radiat. Oncol. 5, 115-133, 1995.

3. F. Khan, R. Potish, Treatment Planning in Radiation Oncology, pp 169-171, William \& Wilkins, Baltimore, 1998.

4. T. R. Mackie, J. Balog, K. Ruchala, D. Shepard, s. Aldridge, E. Fitchard, P. Reckwerdt, G. Olivera, T. McNutt, M. Mehta, “Tomotherapy," Semin Radiat Oncol. 9, 108-17, 1999

5. L. Pisani, D. Lockman, D. Jaffray, D. Yan, A. Martinez, J. Wong, "Setup error in radiotherapy: on-line correction using electronic kilovoltage and megavoltage radiographs," Int. J. Radiat. Oncol. Biol Phys. 47, 825-39, 2000.

6. D. A. Jaffray, D. G. Drake, M. Moreau, A. A.Martinez, J. W. Wong, "A radiographic and tomographic imaging system integrated into a medical linear accelerator for localization of bone and soft-tissue targets, "Int. J. Radiat. Oncol. Biol. Phys. 45, 773-89, 1999.

7. C. L. Hartmann Siantar, R. S. Walling, T. P. Daly, B. Faddegon, N. Albright, P. Bergstrom, A. F. Bielajew, C. Chuang, D. Garrett, R. K. House, D. Knapp, D.-J. Weiczoreck, L. J.Verhey, "Description and dosimetric verification of the Peregrine Monte Carlo dose calculation system for photon beams incident on a water phantom," Med. Phys. 28, $1322-$ 1337, 2001.

8. R. Mohan, "Why Monte Carlo?" Proceedings of the XII International Conference on the Use of Computers in Radiation Therapy, D. D. Leavitt and G. Starkschall, ed. Medical Physics Publishing, Madison, pp 16-18, 1997.

9. L. Wang, C.S. Chui, and M. Lovelock, "A patient-specific Monte Carlo dose-calculation method for photon beams," Med. Phys. 25, 867-878, 1998.

10. J. J. DeMarco, T. D. Solberg, and J. B. Smathers, "A CT-based Monte Carlo simulation tool for dosimetry planning and analysis," Med. Phys. 25, 1-11, 1998.

11. C.-M. Ma, J. S. Li, T. Pawlicki, S.B. Jiamg, J.Deng, "MCDOSE-A Monte Carlo dose calculation tool for radiation therapy treatment planning," XIIIth International Conference on the Use of Computers in Radiation Therapy, Springer, New York, 2000.

12. J. Sempau, S. J. Wilderman, A. F. Bielajew, "DPM, a fast accurate Monte Carlo code optimized for photon and electron radiotherapy treatment planning dose calculations," Phys. med. Biol. 45, 2263-2291, 2000.

13. M. Fippel, "Fast Monte Carlo dose calculations for photon beams based on the VMC electron algorithm," Med. Phys. 26, 1466-1475, 1999.

14. D. A. Jaffray, J. J. Battista, A. Fenster, P. Munro, "X-ray scatter in megavoltage transmission radiography: physical characteristics and influence on image quality," Med. Phys. 21, 45-60, 1994.

15. M. Partridge, P. M. Evans, "The practical implementation of a scatter model for portal imaging at 10 MV," Phys. Med. Biol. 43, 2685-93, 1998.

16. V. N. Hansen, W. Swindell, P. M.. Evans, "Extraction of primary signal from EPIDs using only forward convolution," Med. Phys. 24, 1477-84, 1997.

17. B.M. McCurdy, S. Pistorius, "Photon scatter in portal images: accuracy of a fluence based pencil beam superposition algorithm," Med. Phys. 27, 913-22, 2000.

18. B.M. McCurdy, S. Pistorius, "Photon scatter in portal images: physical characteristics of pencil beam kernels generated using the EGS Monte Carlo code," Med. Phys. 27, 312-20, 2000.

19. L. Spies, M. Partridge, B. A. Groh, T. Bortfeld, "An iterative algorithm for reconstructing incident beam distributions from transmission measurements using electronic portal imaging," Phys. Med. Biol. 46, N203-11, 2001

20. C. Yeboah, S. Pistorius, "Monte Carlo studies of the exit photon spectra and dose to a metal/phosphor portal imaging screen," Med. Phys. 27, 330-9, 2000.

21. B.M. McCurd, S. Pistorius, "A two-step algorithm for predicting portal dose images in arbitrary detectors," Med. Phys. 27, 2109-16, 2000. 
22. T. McNut, T. R. Mackie, P. Reckwerdt, B. R. Paliwal, "Modeling dose distributions from portal dose images using the convolution/superposition method," Med. Phys. 23, 1381-92, 1996.

23. M.-A. Descalle, C.L. Hartmann Siantar, T.B. Yang, C. Chuang, J. Pouliot, "Portal Imaging simulations with PEREGRINE," International Workshop on Monte Carlo Treatment Planning, ed. C. Ma, Stanford, Palo Alto, Nov 9-11, 2000.

24. J. Pouliot, M. Aubin, C. Chuang, B. Pickett, M. Roach, L. Verhey, "Clinical use of an A-Si flat panel for megavoltage portal imaging at UCSF," Med. Phys. 28, 1218, 2001. (abstract)

25. A.E. Schach von Wittenau, P.M. Bergstrom, Jr., and L.J. Cox "Patient-dependent beam-modifier physics in Monte Carlo photon dose calculations," Med. Phys. 27, 935-947, 2000.

26. D. W. O. Rogers, B. A. Faddegon, G. X. Ding, C. M. Ma, J. We, and T. R. Mackie, "BEAM: a Monte Carlo code to simulate radiotherapy treatment units," Med. Phys. 22, 503-524, 1995.

27. L.M. Girouard, J. Pouliot, X. Maldague, A. Zaccarin, "Automatic setup deviation measurements with electronic portal images for pelvic fields," Med. Phys. 25, 1180-1185, 1998.

28. D. Marr and E. Hildreth, "theory of edge detection," Proc. Roy. Soc. London, Ser. B207: 187-217, 1980.

29. G. Glutchev and S. Shalev, "Fast algorithm for radiation field edge detection," SPIE Image Processing 1898, 296-303, 1993.

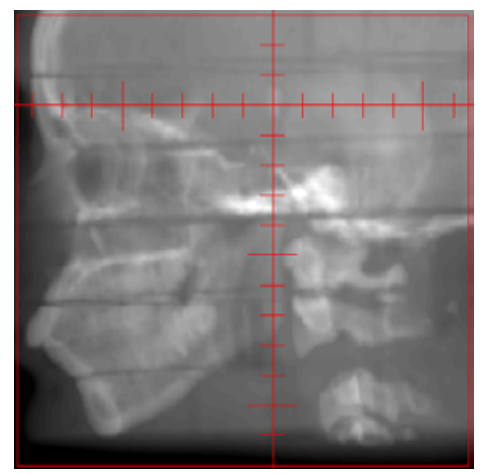

a)

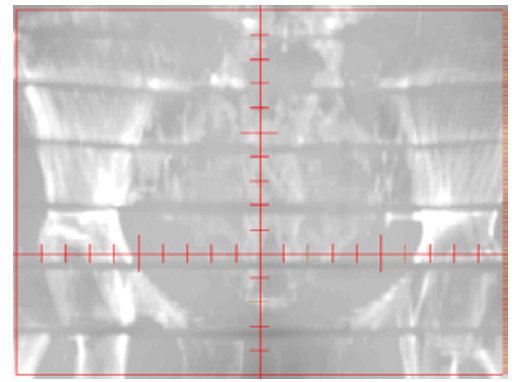

d)

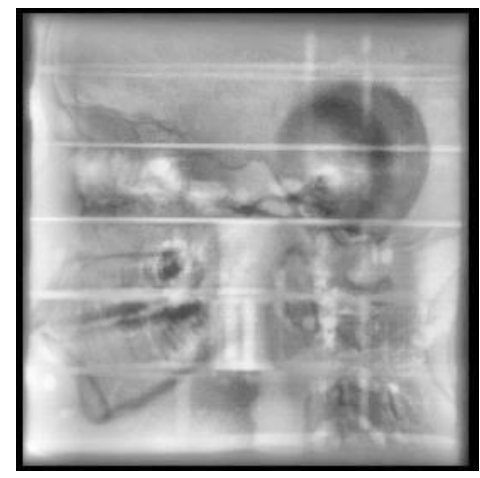

c)

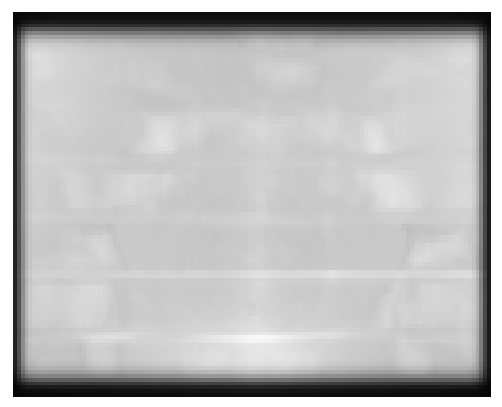

e)

f)

Figure 1: Portal images of the Rando phantom for the Head and Pelvic sections; we show a) the DRR generated from CT scans, b) the clinical portal image, and c) the PEREGRINE simulated image for the Head section and, correspondingly, d), e), and f) for the Pelvic section.

Table 1: Rando phantom comparison of applied and detected set-up deviation using PEREGRINE simulated image as the reference image

\begin{tabular}{|c|c|c|c|c|c|c|c|}
\hline \multirow{2}{*}{$\begin{array}{c}\text { Rando } \\
\text { phantom } \\
\text { Section }\end{array}$} & $\begin{array}{c}\text { Applied } \\
\text { Displacement }\end{array}$ & \multicolumn{2}{|c|}{ Total Deviation (mm) } & $\begin{array}{c}\text { Corrected Deviation } \\
(\mathrm{mm})\end{array}$ & \multicolumn{2}{|c|}{$\Delta(\mathrm{mm})$} \\
\cline { 3 - 8 } & & $\mathrm{x}$ & $\mathrm{y}$ & $\mathrm{x}$ & $\mathrm{y}$ & $\mathrm{x}$ & $\mathrm{y}$ \\
\hline
\end{tabular}




\begin{tabular}{|c|l|c|c|c|c|c|c|}
\hline \multirow{4}{*}{ HEAD } & None & 4.7 & 0.6 & - & - & - & - \\
\cline { 2 - 7 } & Anterior 7 mm & 11.3 & 0.0 & 6.6 & -0.6 & 0.4 & 0.6 \\
\cline { 2 - 7 } & $\begin{array}{l}\text { Anterior 7 mm } \\
\text { Caudal } 8 \mathrm{~mm}\end{array}$ & 10.7 & -7.3 & 6.0 & -7.9 & 1.0 & 0.1 \\
\hline \multirow{5}{*}{ PELVIS } & None & 1.0 & 15.0 & - & - & - & - \\
\cline { 2 - 8 } & Left Lateral 7 mm & -5.5 & 15.0 & -6.5 & 0.0 & 0.5 & 0.0 \\
\cline { 2 - 8 } & Right Lateral 8 mm & 9.5 & 15.0 & 8.5 & 0.0 & 0.5 & 0.0 \\
\cline { 2 - 8 } & $\begin{array}{l}\text { Right Lateral 8 mm } \\
\text { Caudal 8 mm }\end{array}$ & 8.5 & 9.0 & 7.5 & 6.0 & 0.5 & 2.0 \\
\hline
\end{tabular}

QuickTime $^{T M}$ and a

GIF decompressor
are needed to see this picture.

a) No displacement

$\mathrm{mm}$ Anterior $8 \mathrm{~mm}$ Caudal
QuickTime ${ }^{T M}$ and a

GIF decompressor
are needed to see this picture

b) Displacement 7 
Figure 2: Correlation of portal images and PEREGRINE simulated images for a Rando phantom. In a) the reference image (PEREGRINE) is in the bottom right corner. The test image (clinical) is in the bottom left corner. In the top right corner, the two image contours are superposed before correlation. Finally, in the top left corner, the contours overlap after the correlation correction was applied. Images in a) and b) are for the Head section. Correspondingly, images in c) and d) are for the Pelvis section.

Table 2: Left lateral beam Prostate treatment plan comparison of applied and detected set-up deviation using PEREGRINE simulated image as the reference image

\begin{tabular}{|l|l|l|l|l|l|l|}
\hline \multicolumn{2}{|l|}{ Day } & 1 & 2 & 3 & 4 & 5 \\
\hline $\begin{array}{l}\text { Total Deviation } \\
(\mathrm{mm})\end{array}$ & $\mathrm{X}$ & -8.1 & -9.5 & -12.2 & -8.8 & -9.5 \\
\cline { 2 - 7 } & $\mathrm{Y}$ & -0.5 & -5.2 & -1.6 & 2.1 & -2.1 \\
\hline
\end{tabular}
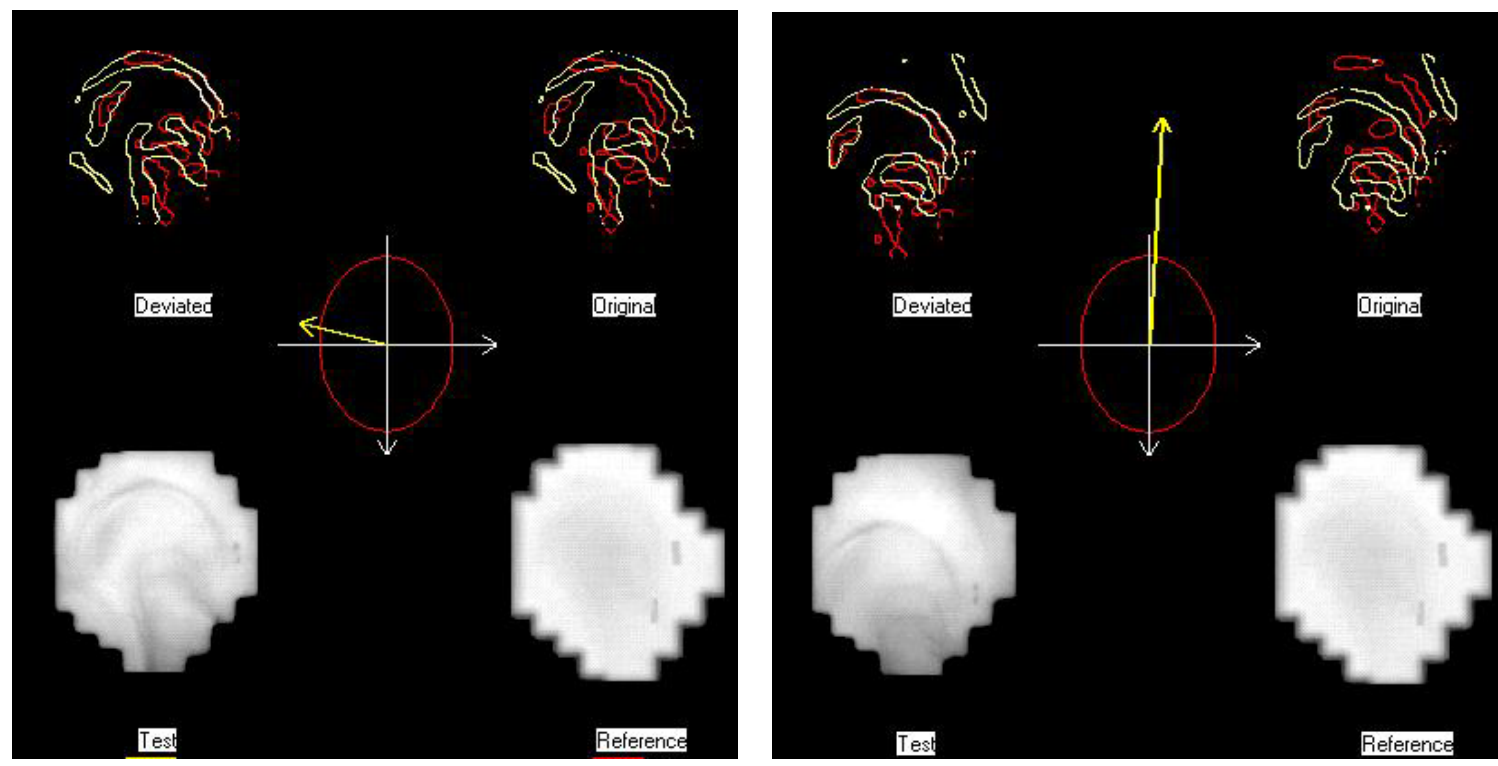

a)

b)

Figure 3: Left lateral beam, 7-field prostate case. Detection of set-up deviations using the PEREGRINE image as the reference image are in a) for small displacement and in b) for large displacement. Images within a) and b) are as explained in Figure 2. 